\title{
Influence of Geometric Parameters on Characteristics of an Interdigital Dielectrometry Sensor
}

\author{
A.V. Mamishev M. Zahn B.C. Lesieutre B.A. Berdnikov \\ Department of Electrical Engineering and Computer Science \\ Massachusetts Institute of Technology \\ Cambridge, MA 02139, USA
}

\begin{abstract}
Interdigital dielectrometry technology provides means for non-destructive measurement of various physical properties in the bulk of insulating materials. Results of continuing work on the refinement of this technology are presented. A close match of theoretical results and experimental values is achieved with a sensor designed to minimize non-ideal effects.

Optimization of the sensor design and parameter estimation algorithms is performed by studying the influence of various geometric parameters on the sensor's response.
\end{abstract}

\section{Introduction}

Interdigital electrodes are currently used as emitting electrodes for modulation of surface acoustic waves [1], as capacitive electrodes in position detectors, and for other applications. The theoretical foundation for the use of interdigital electrodes for characterizing of properties of multilayered materials was developed in the eighties [2].

The first practical implementations of the multiwavelength sensors exhibited noticeable differences between the theoretical predictions and the measurements of interelectrode impedances. When the inverse problem of material characterization is being solved, relatively small discrepancies can lead to large errors.

The goal of this study is to make sure that the forward problem of finding the equivalent circuit parameters is solved with high accuracy and confidence. A sensor designed to reduce non-ideal effects is presented and analyzed below.

\section{Sensor Design}

Overall design. The overall design of the sensor is shown in Figure 1. The flexible sensor consists of three sets of electrodes deposited on a common flexible substrate. Teflon (dielectric permittivity is equal to 2.1) was chosen for the substrate because of its low moisture absorption characteristics, which eliminates the need for a moisture barrier coating. The sensing electrodes are shielded by guard electrodes driven by a follower operational amplifier. The gray shade under the electrodes indicates individual guard planes on the back of the substrate, which are driven at guard voltage of the corresponding sensing electrode. The advantage of such design versus use of a common ground plane is discussed in [3].

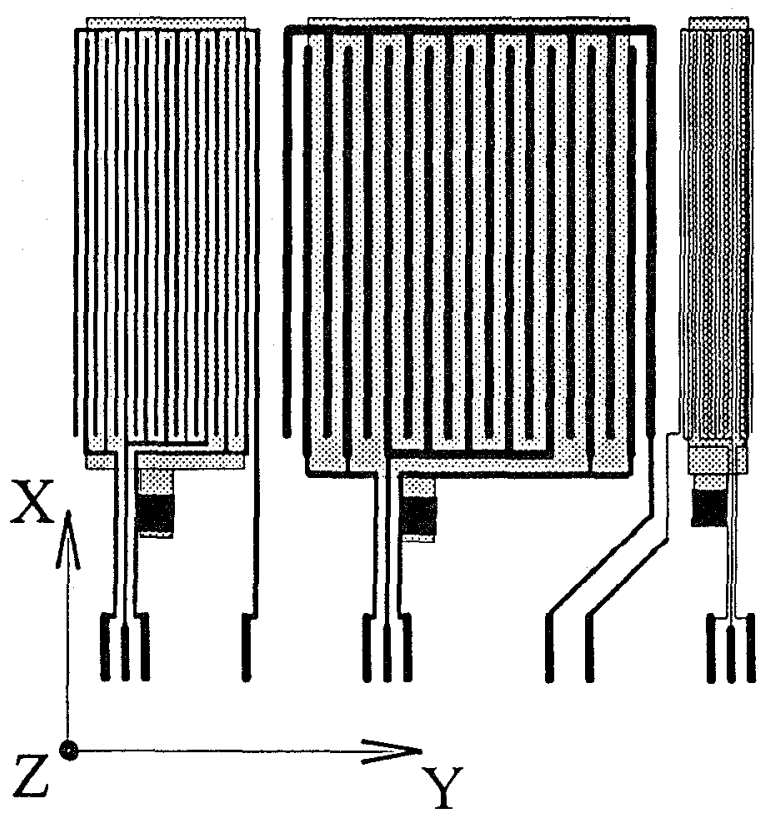

Figure 1. The three-wavelength interdigital sensor. 
Circuit model. The equivalent circuit model of each individual wavelength is shown in Figure 2. Together with the interface, the interdigital structure creates a resistive-capacitive divider, the output of which is recorded and analyzed.

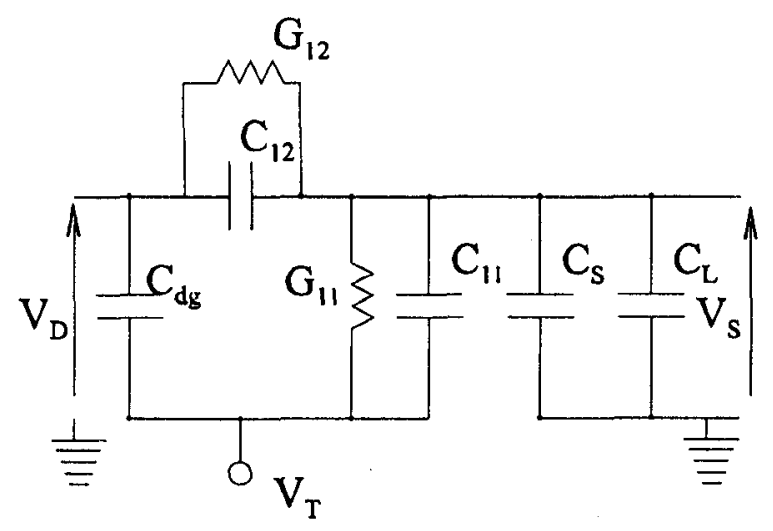

Figure 2. The equivalent circuit model of the sensor.

The ground potential of the circuit is defined through a lumped load capacitor $C_{L}$, which needs to be sufficiently large to mitigate variations of the stray capacitance $C_{S}$ from the sensing electrode to the ambient objects. However, if the value of $C_{L}$ is too large (over $300 \mathrm{pF}$ for the present geometry), the sensing signal becomes too low, comparable to the noise levels.

The sensor operates with $V_{T}=V_{S}$. The voltage measured in the sensing electrodes can be expressed as:

$$
G=\frac{V_{S}}{V_{D}}=\frac{G_{12}+j \omega C_{12}}{G_{12}+j \omega C_{12}+j \omega\left(C_{L}+C_{S}\right)},
$$

where $V_{S}$ is a voltage on a sensing electrode and $V_{D}$ is the voltage on the driven electrode.

This circuit can be solved directly for $\mathrm{G}_{12}$ and $\mathrm{C}_{12}$, which can be mapped to the permittivity $\varepsilon$ and the conductivity $\sigma$ of the test dielectric [3].

Motivation. The idealized model [2] requires the sensor be infinitely long and wide. A large number of finger pairs which are very long with respect to the spatial wavelength of the sensor (distance between the two adjacent fingers of the equal potential) would provide adequate approximation. The expected penetration depth of the sensor to be used for monitoring of moisture dynamics of the transformer pressboard is on the order of one millimeter. It was demonstrated in a previous study [4], that the spatial wavelength of the sensor must be about four times the penetration depth. Given such dimensions, and that several distinct interdigitated structures must be present, the sensor must be relatively large in order to be reasonably approximated with the idealized model.

The particular design of the three-wavelength sensor used in this study has several features which help to reduce the difference between the theoretical model and the actual measurements for cases when only a small number of fingers can be used.

\section{Measurement of Interelectrode Capacitances}

End effects. Two types of end discontinuities must be considered: the finite number of fingers along the axis $\mathrm{Y}$, and the finite length of the electrodes along the axis $X$. The effect of the former is alleviated by adding additional guard fingers on both sides of each wavelength, as shown in Figure 1.

Evaluation of the end effects alcing the axis $X$ can be accomplished in several ways. Closed-form solutions as well as empirical models of interdigital sensor discontinuities had been developed by several authors, for example, see [5]. At the same time, a precise geometry of the end areas usually differs the idealized models. A simple experimental procedure was performed in order to approximate the equivalent capacitance of the end area.

The geometry of the end area of the $5 \mathrm{~mm}$ wavelength is shown in Figure 3. A piece of Lexan (GE polycarbonate) was placed above the sensor so that the $\mathrm{Y}-\mathrm{Z}$ plane (as defined by Figure 1) containing the airLexan boundary goes through the zero $\mathrm{X}$ in Figure 3 . The Lexan sample was then repositioned in $0.25 \mathrm{~mm}$ increments in the direction of regative $X$, so that it gradually covers the electrode. The relative permittivity of Lexan is 3.17. Naturally, the equivalent capacitance $\mathrm{C}_{12}$ starts changing as the specimen moves along the axis. The change in capacitance during this measurement is shown in Figure 4. The growth becomes linear when the specimen reaches the linear part of the electrodes. The equivalent meander length of the end area can now be found by subtracting the output at the point $-3.125 \mathrm{~mm}$ and at $0 \mathrm{~mm}$ and relating it to the linear function at the subsequent coordinates. After taking the enc effects into account, the meander lengths were found to be $50+\lambda / 0.8 \mathrm{~cm}$ for each wavelength. 


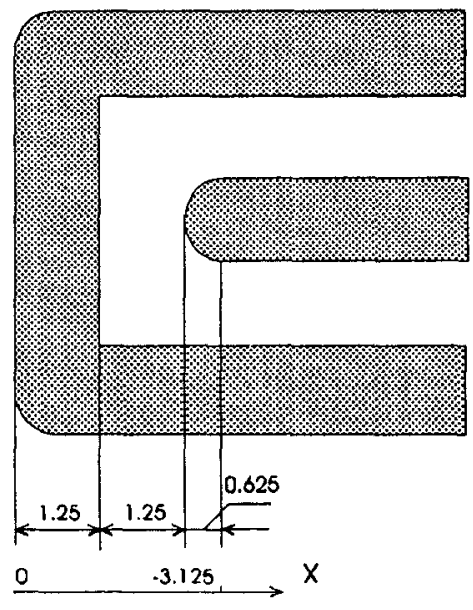

Figure 3. End area of the individual finger of the 5.0 $\mathrm{mm}$ wavelength sensing electrode.

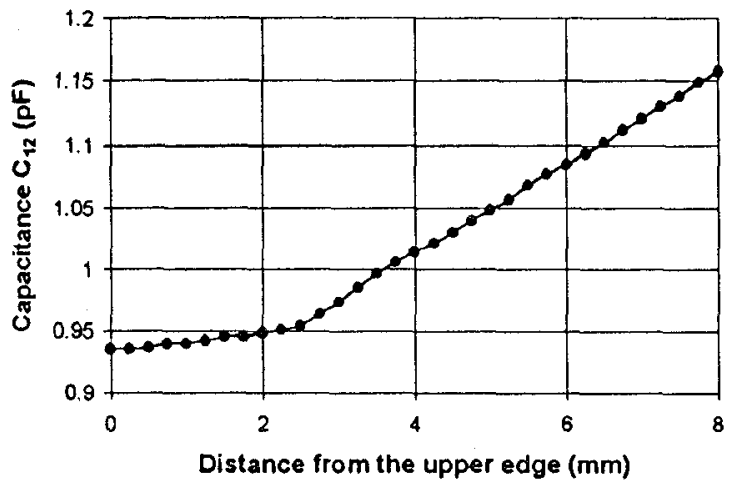

Figure 4. Sensor's response to the linear movement of the test specimen.

Cross-coupling. A slight dependence of the ouput of one wavelength on the voltage of the others was observed in some cases. There are two ways to perform dielectrometry measurements with a multiwavelength sensor, either with all driven electrodes energized simultaneously, or by doing separate measurements with each wavelengths while holding all other electrodes on the substrate at the guard potential of the active sensing electrode. The difference in the output can be anywhere from a few percent to more than 100 percent depending on the type of experiment. Measurements in air result in very low cross-coupling, yet traceable given our precision requirements. In general, errors due to cross-coupling are larger when highly conductive materials are studied.
Comparison of theory and measurements. The capacitance of each wavelength was measured at a frequency of $1 \mathrm{kHz}$ using both modes, as well as computed with FEM software (Ansoft). Real geometrical parameters were used in the simulation. The results of measurements and calculations are presented in Table 1. The last three lines in this table show the maximum accuracy which currently can be achieved. The difference between the theory and the measurements does not exceed $5 \%$.

Table 1. Comparison of calculated and measured values of the interelectrode capacitance in air.

\begin{tabular}{|l|c|c|c|}
\hline Wavelength & $\lambda=1 \mathrm{~mm}$ & $\lambda=2.5 \mathrm{~mm}$ & $\lambda=5.0 \mathrm{~mm}$ \\
\hline Metallization ratio & 0.396 & 0.469 & 0.476 \\
\hline $\begin{array}{l}\text { Thickness of Electrodes, } \\
\mu \mathrm{m}\end{array}$ & 14 & 14 & 14 \\
\hline $\begin{array}{l}\mathrm{C}_{12}(\mathrm{pF}), \text { Measurement, } \\
\text { with Cross-Coupling }\end{array}$ & 2.695 & 1.489 & 0.986 \\
\hline $\mathrm{C}_{12}(\mathrm{pF})$ FEM (Ansof) & 2.769 & 1.591 & 0.895 \\
\hline $\begin{array}{l}\mathrm{C}_{12}(\mathrm{pF}), \text { Measurement, } \\
\text { without Cross-Coupling }\end{array}$ & 2.663 & 1.536 & 0.935 \\
\hline Mismatch (\%) & 3.8 & 3.5 & 4.5 \\
\hline
\end{tabular}

\section{Sensitivity to the Variations in Geometry}

Variations of geometry within the production tolerances of the manufacturer result in noticeable changes in the parameters of an interdigital sensor. For example, the metallization ratio (ratio of copper covered area to the total area of the sensor) in Table 1 should ideally be equal to 0.5 . The curves presented in this section were obtained by finite-element modeling, and can be used to evaluate the error introduced by variations in the geometry of the sensor. The parasitic capacitance, defined as the difference of capacitance of the electrodes of given thickness and zero thickness electrodes, is practically a linear function of thickness, as can be seen in Figure 4. Also, it has been previously shown that the parasitic capacitance is a linear function of dielectric permittivity of the specimen [6]. Dependence of the interelectrode capacitance on the metallization ratio for the $5.0 \mathrm{~mm}$ wavelength is shown in Figure 6. The relation is similar for the other wavelengths when normalized with respect to size and capacitance. 


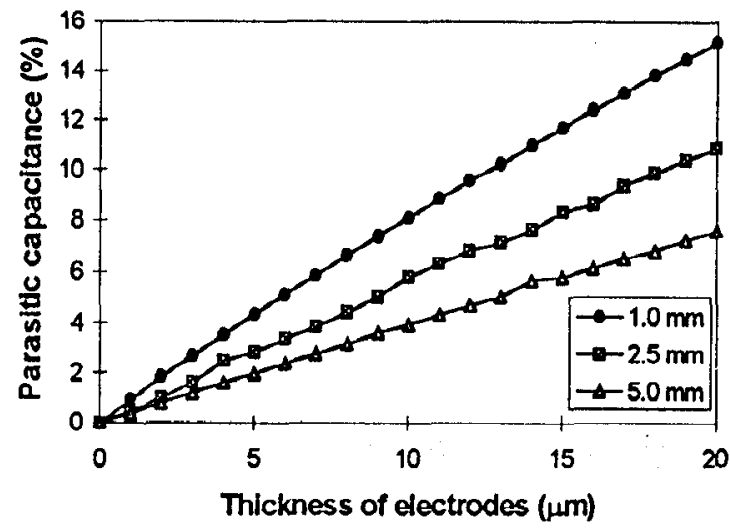

Figure 5. Percent error introduced by parasitic capacitance to the sensor output.

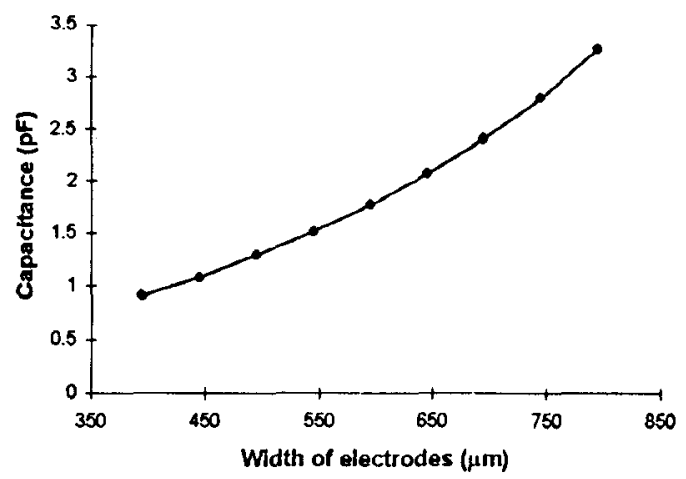

Figure 6. Dependence of interelectrode capacitance $C_{12}$ on the width of the electrodes $(5.0 \mathrm{~mm}$ wavelength, per meter length).

\section{Conclusions}

Analysis of a three-wavelength sensor designed for precise dielectrometry measurements is presented. Sensitivity due to variations in the geometric parameters is estimated. A close match (within 5\%) between the theoretical predictions and measurements is achieved. The paper provides benchmark data which serves as basis for various dielectrometry measurements.

\section{Acknowledgments}

The authors would like to acknowledge the support of the Electric Power Research Institute, under grant WO 3334-1, managed by Mr. S. Lindgren, and the National
Science Foundation under grant No. ECS-9523128. The donation of Maxwell soltware by Ansoft Corp. is gratefully appreciated. The authors would like to thank Mr. Darrell Schlicker, and Ms. Yanqing Du, both from the MIT Laboratory for Electromagnetic and Electronic Systems, for valuable discussions and assistance with the experimerits.

\section{References}

[1] R.T. Kollipara, A.S. Mohammed, T.K. Plant, and V.K. Tripathi, "Modeling and Design of Interdigital Structures," IEEE Transactions on Electron Devices, vol. 38, no. 11, November 1991, pp. 2575-77.

[2] M.C. Zaretsky, L. Mouayad, and J.R. Melcher, "Continuum Properties frorn Interdigital Electrode Diclectrometry," IEEE Transactions on Electrical Insulation, vol. 23, no.6, December 1988, pp. 897917.

[3] A.V. Mamishev, M. Zahn, "Techniques for SemiEmpirical Characterization of Material and Sensor Properties in Interdigital Dielectrometry," 1996 IEEE International Symposium on Electrical Insulation, Montreal, Canada, July 1996, pp. 53-56.

[4] A.V. Mamishev, Y. Du, M. Zahn, "Measurement of Dielectric Property Distributions Using Interdigital Dielectrometry Sensors," IEEE Conference on Electrical Insulation and Dielectric Phenomena, Virginia Beach, VA, October 1995, pp. 309-312.

[5] M. Kirschning and R.H. Jansen, "Accurate, Wide Range Design Equations for the Frequency Dependent Characteristics of Parallel Coupled Microstrip Lines", IEEE Transactions on Microwave Theory Tech., vol. MTT-32, pp. 83-90, January 1984.

[6] P.A. von Guggenberg, and J.R. Melcher, "Application of Interdigital Dielectrometry to Moisture and Double Layer Measurements in Transformer Insulation", Ph. D. thesis, EECS Department, MIT, 1993.

[7] A. K. Jonscher, Dielectric Relaxation in Solids, Chelsea Dielectric Press, London, 1983.

[8] Y. K. Sheiretov, M. Zahn, "Dielectrometry Measurements of Moisture Dynamics in OilImpregnated Pressboard," 1594 IEEE International Conference on Properties and Applications of Dielectric Materials, University of Queensland, Brisbane, Australia, July 1994 